\title{
Uncertainties of Thermographic Measurements - Exercises
}

\author{
S. Dudzik ${ }^{1}, \underline{\text { W. Minkina }}{ }^{1}$ \\ ${ }^{1}$ Częstochowa University of Technology, Faculty of Electrical Engineering, \\ Institute of Optoelectronics and Measurement Systems \\ Częstochowa, Al. Armii Krajowej 17, 42-200 \\ minkina@el.pcz.czest.pl
}

\begin{abstract}
In the contemporary metrological sciences there are two main theories. The theory of measurement errors and the measurement uncertainty theory. Both of them were described in [1] with respect of the analysis of the thermographic measurements accuracy. However, the practical examples for the measurement errors and uncertainties estimation were not included in the abovementioned monograph. Therefore, the practical examples of error calculation were presented in [2]. The purpose of this paper is to show the exercises of estimation of the combined standard uncertainty and its components occurred in the non-contact temperature measurements made by means the infrared cameras. In this work the calculations conducted at the most common conditions existed in practical situations were presented. In each example the $95 \%$ coverage intervals were calculated. They enable the universal accuracy estimation in accordance with the guidelines of the international metrological organizations [3, 4]. This paper is also an extension of the material presented in works [5, 6].
\end{abstract}

Key words: combined standard uncertainty, infrared cameras, thermographic measurements.

\section{Analysis of the measurements accuracy in the presence of the random interactions}

In this work the static model of thermographic measurement is presented. The static model (i.e. the model in which a detector integration time is long enough compared with a time constant of infrared detector) can be expressed as a function of five parameters:

$T_{o b}=f\left(\varepsilon, T_{0}, T_{a t m}, \omega, d\right)$

where: $T_{o b}$ - object temperature $(\mathrm{K}), \varepsilon$ - band emissivity of the object surface, $T_{0}-$ ambient temperature (K), $T_{\text {atm }}$ - temperature of atmosphere $(\mathrm{K}), \omega$ - relative humidity, $d-$ camera-to-object distance $(\mathrm{m})$.

In equation (1) the model of the atmosphere transmission can be calculated using a complex approximated functions delivered by a FLIR company [7]. Then, the model of atmospheric transmission can be described as the following simplified formula:

$$
P_{a t m}=f\left(\omega, d, T_{a t m}\right),
$$

where: $P_{a t m}$ - an atmospheric transmission coefficient.

In practice, the measurement uncertainty can be defined as [4]:

$$
u\left(x_{i}\right)=\sqrt{\frac{1}{N-1} \sum_{i=1}^{N}(x-\bar{x})^{2}},
$$

where: $x_{i}$ - the result of a single measurement, $N$ - the number of measurements.

Additionally, when we will assume a lack of the correlations between the input variables of measurement model, we can define the combined standard uncertainty, described using the following formula:

$u_{c}^{2}(y)=\sum_{i=1}^{N}\left(\frac{\partial f}{\partial x_{i}}\right)^{2} u^{2}\left(x_{i}\right)$,

where $f\left(x_{1}, x_{2}, \ldots x_{n}\right)$ is a measurement model.

The expanded uncertainty define the limits of the coverage interval at the given level of confidence, the most commonly at $95 \%$. The expanded uncertainty can be expressed as the following equation:

$$
U=k u_{c}(y),
$$

where: $k$ - the expansion coefficient.

Unfortunately, in most cases, the probability distribution of the output variable of measurement model remains unknown. Therefore, the Joint Committee for Guides in 
Metrology (JCGM) developed the document [4], in which, the method of the propagation of distributions is featured. This method allows for correct estimation of accuracy including the following cases:

- The partial derivatives are countless.

- The probability distribution of the output variable is not Gaussian.

- The measurement model is strongly nonlinear.

The evaluation of the measurement uncertainty with the method for the propagation of distributions can be divided into the following stages [4]:

- define of the measurand (the output quantity of the measurement)

- define of the input variables of the measurement model,

- design of the measurement model,

- determination of the probability distributions of input variables,

- evaluation of the probability distribution of the output variable (measurand),

- estimation of the statistical parameters of the probability distribution of the output variable (e.g. 95\% coverage interval).

The software used in the numerical calculations of the accuracy

In the examples of the numerical calculations, the programs created in the MATLAB environment were used. The basic functions of the software are [1]:

- generating of the random variable realizations with the parameters and distributions defined by the user,

- reading of the calibration parameters and the reference values from the Agema File Format files,

- simulation of the data processing algorithm based on the mathematical model of the infrared camera measurement,

- estimation of the combined standard uncertainty and its components,

- estimation of the $95 \%$ coverage intervals,

- graphical presentation of the simulation results.

The screenshot of the main window of the program used in the abovementioned examples is presented in fig. 1. The source code of this software was attached to the work [1].

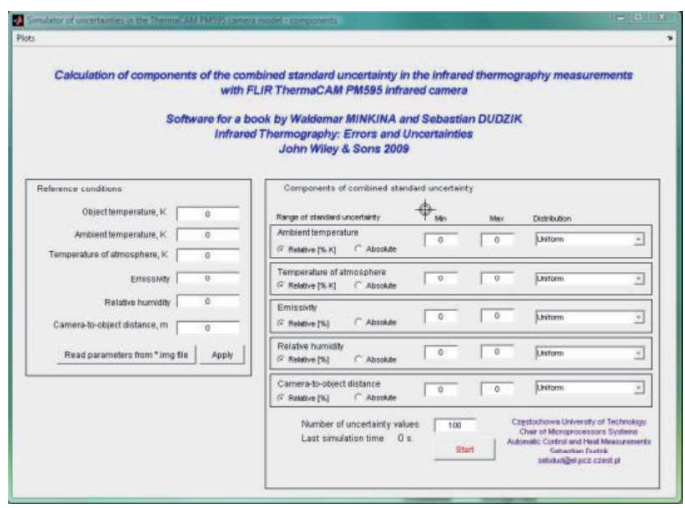

Fig. 1. The main window screen shot of the program used for the calculations of the components of combined standard uncertainty in the measurements with the FLIR ThermaCAM PM595 infrared camera.

\section{Exemplary numerical calculations of the components of combined standard uncertainty in temperature measurements carried out with the FLIR infrared cameras}

\section{Exercise 1}

The temperature measurement was conducted using the FLIR ThermaCAM PM595 infrared camera. The emissivity of the investigated object was equal to $\varepsilon_{o b}=0,4$. Calculate the component of the relative combined standard uncertainty $u\left(T_{o b}\right)$, associated with the object emissivity $\varepsilon_{o b}$, assuming that $T_{o b}=303 \mathrm{~K}(30$ ${ }^{\circ} \mathrm{C}$ ), if we know that the relative standard uncertainty $u\left(\varepsilon_{o b}\right)=10 \%$. The estimates of the remaining input variables of the model (1) should be assumed according to the table 1 .

Tab. 1: The values of the input variables of the model (1) assumed for the calculations of the accuracy

\begin{tabular}{|c|c|c|c|c|}
\hline$\varepsilon_{o b}$ & $\begin{array}{c}T_{o} \\
(\mathrm{~K})\end{array}$ & $\begin{array}{c}T_{a t m} \\
(\mathrm{~K})\end{array}$ & $\omega$ & $\begin{array}{c}d \\
(\mathrm{~m})\end{array}$ \\
\hline 0,4 & 293 & 293 & 0,5 & 1,0 \\
0,9 & & \\
\hline
\end{tabular}

\section{Solution}

To solve the task from the exercise 1, the abovementioned program was used. The simulation results (i.e. the components of the combined standard uncertainty associated with the object emissivity $\varepsilon_{o b}$ ) were presented in Fig. 2.

The value of the component of the relative combined standard uncertainty associated with the object emissivity, for the relative standard uncertainty of the object emissivity $u\left(\varepsilon_{o b}\right)=10 \%$, and the object emissvity $\varepsilon_{o b}=0,4$, can be read from Fig. 2. For the object temperature $T_{o b}=$ $303 \mathrm{~K}\left(30^{\circ} \mathrm{C}\right)$, it is equal to $u\left(T_{o b}\right) \approx 0,4 \%$. 


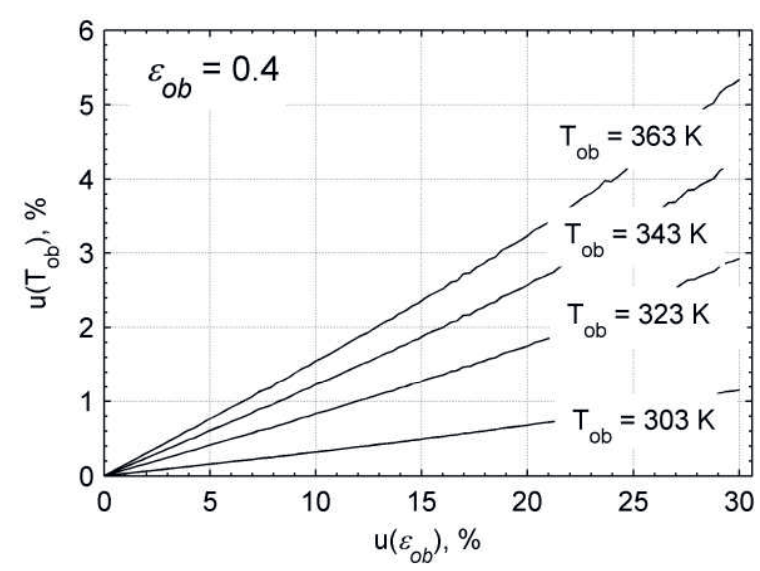

Fig. 2. Component of the relative combined standard uncertainty associated with emissivity $\varepsilon_{o b}$. Results of simulations for data collected in tab. 1.

\section{Exercise 2}

The temperature measurement was conducted using the FLIR ThermaCAM PM595 infrared camera. The emissivity of the investigated object was equal to $\varepsilon_{o b}=0,9$. Calculate the component of the relative combined standard uncertainty $u\left(T_{o b}\right)$, associated with the object emissivity $\varepsilon_{o b}$, assuming that $T_{o b}=363 \mathrm{~K}(90$ $\left.{ }^{\circ} \mathrm{C}\right)$, if we know that the relative standard uncertainty $u\left(\varepsilon_{o b}\right)=20 \%$. The estimates of the remaining input variables of the model (1) should be assumed in accordance with the table 1.

\section{Solution}

The component of the relative combined standard uncertainty associated with the object emissivity $\varepsilon_{o b}$ were presented in Fig. 3. They were obtained from simulations conducted for data from table 1.

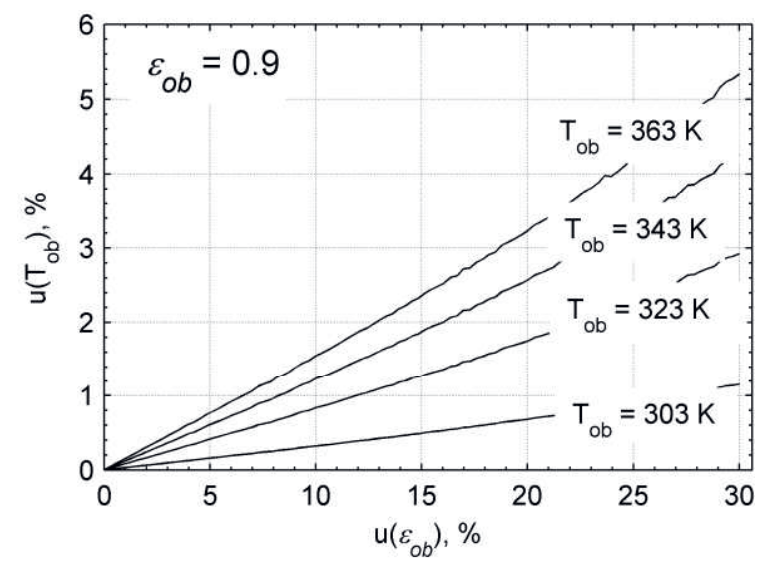

Fig. 3. Component of the relative standard uncertainty associated with emissivity $\varepsilon_{o b}$. Results of simulations for data collected in tab. 1.

As you can see in Fig. 3, the value of the component of the relative combined standard uncertainty for $u\left(\varepsilon_{o b}\right)=20 \%, \varepsilon_{o b}=0,9$ and $T_{o b}=$ $303 \mathrm{~K}\left(30^{\circ} \mathrm{C}\right)$, equals to $u\left(T_{o b}\right) \approx 0,4 \%$.

\section{Exercise 3}

The temperature measurement was conducted using the FLIR ThermaCAM PM595 infrared camera. The emissivity of the investigated object was equal to $\varepsilon_{o b}=0,4$. Calculate the component of the relative combined standard uncertainty $u\left(T_{o b}\right)$, associated with the ambient temperature $T_{o}$, assuming that $T_{o b}=303 \mathrm{~K}(30$ $\left.{ }^{\circ} \mathrm{C}\right)$, if we know that the relative standard uncertainty $u\left(T_{o}\right)=0,5 \%$. The values of the remaining input variables of the model (1) should be assumed according to the table 1 .

\section{Solution}

To solve the task from the exercise 3 , the simulations with data from table 1 were carried out. The component of the relative combined standard uncertainty associated with the ambient temperature $T_{o}$ obtained from the simulations were presented in Fig. 4.

The value of the component of the relative combined standard uncertainty associated with the ambient temperature $T_{o}$, for the relative standard uncertainty of the ambient temperature $u\left(T_{o}\right)=0,5 \%$, and the object emissvity $\varepsilon_{o b}=0,4$ can be read from Fig. 4 . For the object temperature $T_{o b}=303 \mathrm{~K}\left(30^{\circ} \mathrm{C}\right)$, the value of the component $u\left(T_{o b}\right) \approx 0,75 \%$.

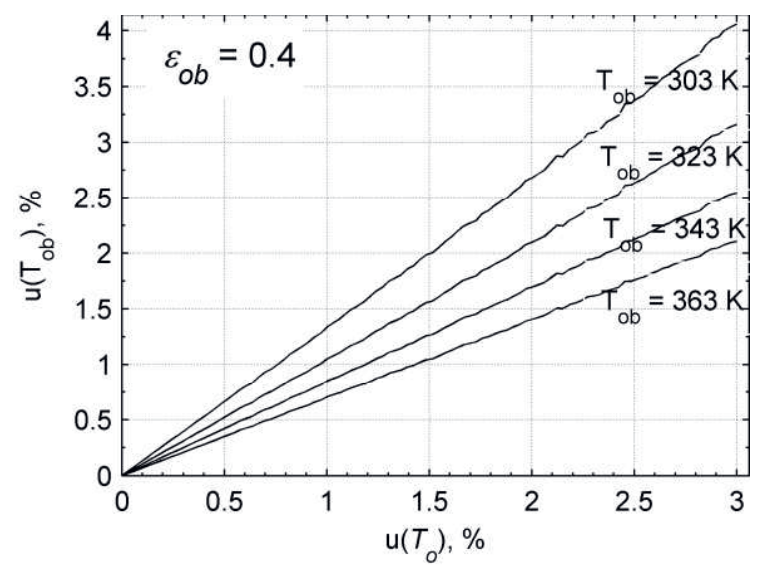

Fig. 4. Component of the relative standard uncertainty associated with the ambient temperature $T_{o}$. Results of simulations for data collected in tab. 1 .

\section{Exercise 4}

The temperature measurement was conducted using the FLIR ThermaCAM PM595 infrared camera. The emissivity of the investigated object was equal to $\varepsilon_{o b}=0,9$. Calculate the component of the relative combined standard uncertainty $u\left(T_{o b}\right)$, associated with the ambient temperature $T_{o}$, assuming that $T_{o b}=363 \mathrm{~K}(90$ ${ }^{\circ} \mathrm{C}$ ), if we know that the relative standard uncertainty $u\left(T_{o}\right)=2 \%$. The values of the remaining input variables of the model (1) should be assumed according to the table 1 . 


\section{Solution}

To solve the task from the exercise 4 , the simulations with data from table 1 were carried out. The components of the combined standard uncertainty associated with the ambient temperature $T_{o}$ obtained from the simulations were presented in Fig. 5.

The value of the component of the relative combined standard uncertainty associated with the ambient temperature $T_{o}$, for the relative standard uncertainty of the ambient temperature $u\left(T_{o}\right)=2 \%$, and the object emissvity $\varepsilon_{o b}=0,9$ can be read from Fig. 5 . For the object temperature $T_{o b}=363 \mathrm{~K}\left(90^{\circ} \mathrm{C}\right)$, the value of the component $u\left(T_{o b}\right) \approx 0,11 \%$.

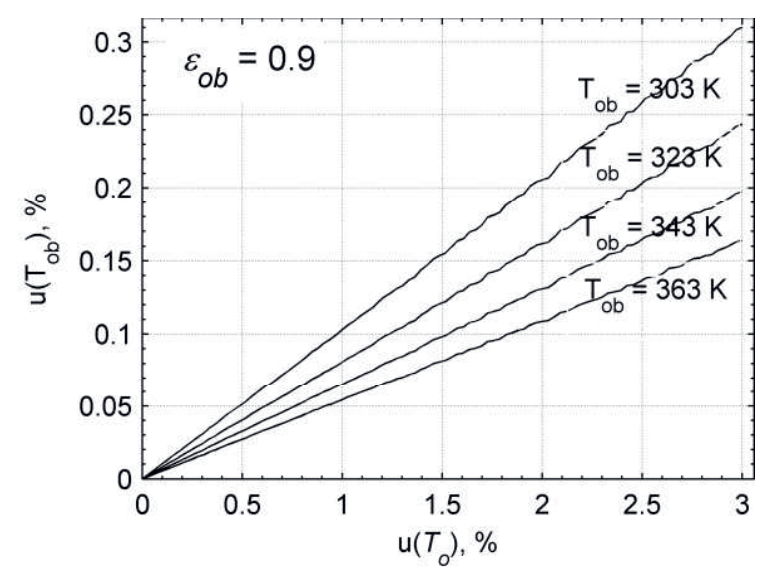

Fig. 5. Component of the relative standard uncertainty associated with the ambient temperature $T_{0}$. Results of simulations for data collected in tab. 1.

\section{Exercise 5}

The temperature measurement was conducted using the FLIR ThermaCAM PM595 infrared camera. The emissivity of the investigated object was equal to $\varepsilon_{o b}=0,4$. Calculate the component of the relative combined standard uncertainty $u\left(T_{o b}\right)$, associated with the camerato-object distance $d$, assuming that $T_{o b}=303 \mathrm{~K}$ $\left(30{ }^{\circ} \mathrm{C}\right)$, if we know that the relative standard uncertainty $u(d)=10 \%$. The values of the remaining input variables of the model (1) should be assumed according to the table 1 .

\section{Solution}

To solve the task from the exercise 5 , the simulations with data from table 1 were carried out. The component of the relative combined standard uncertainty associated with the camera-to-object distance $d$ obtained from the simulations were presented in Fig. 6 .

The value of the component of the relative combined standard uncertainty associated with the camera-to-object distance $d$, for the relative standard uncertainty of the camera-to-object distance $u(d)=10 \%$, and the object emissivity $\varepsilon_{o b}=0,4$ can be read from Fig. 6 . For the object temperature $T_{o b}=303 \mathrm{~K}\left(30{ }^{\circ} \mathrm{C}\right)$, the value of the component $u\left(T_{o b}\right) \approx 0,0010 \%$.

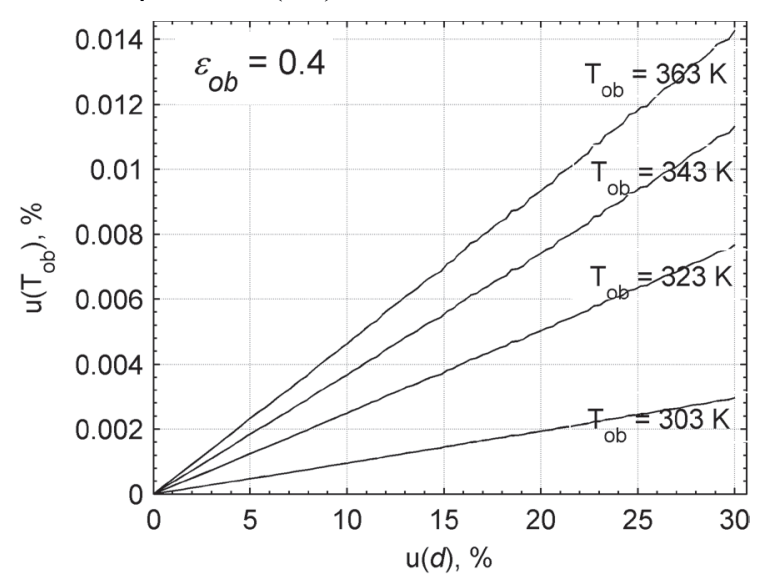

Fig. 6. Component of the relative standard uncertainty associated with the camera-to-object distance $d$. Results of simulations for data collected in tab. 1.

\section{Exercise 6}

The temperature measurement was conducted using the FLIR ThermaCAM PM595 infrared camera. The emissivity of the investigated object was equal to $\varepsilon_{o b}=0,9$. Calculate the component of the relative combined standard uncertainty $u\left(T_{o b}\right)$, associated with the camerato-object distance $d$, assuming that $T_{o b}=363 \mathrm{~K}$ $\left(90{ }^{\circ} \mathrm{C}\right)$, if we know that the relative standard uncertainty $u(d)=20 \%$. The values of the remaining input variables of the model (1) should be assumed according to the table 1 .

\section{Solution}

To solve the task from the exercise 6 , the simulations with data from table 1 were carried out. The component of the relative combined standard uncertainty associated with the camera-to-object distance $d$ obtained from the simulations were presented in Fig. 7 .

The value of the component of the relative combined standard uncertainty associated with the camera-to-object distance $d$, for the relative standard uncertainty of the camera-to-object distance $u(d)=20 \%$, and the object emissivity $\varepsilon_{o b}=0,9$ can be read from Fig. 7. For the object temperature $T_{o b}=363 \mathrm{~K}\left(90^{\circ} \mathrm{C}\right)$, the value of the component $u\left(T_{o b}\right) \approx 0,0095 \%$.

\section{Exemplary numerical calculations of the combined standard uncertainty and $95 \%$ coverage interval in temperature measurements carried out with the FLIR infrared cameras}

\section{Exercise 7}

The temperature measurement was conducted using the FLIR ThermaCAM PM595 infrared camera. The emissivity of the investigated 
object was equal to $\varepsilon_{o b}=0,9$. Calculate the combined standard uncertainty $u_{c}\left(T_{o b}\right)$ and evaluate the $95 \%$ coverage interval $I_{95 \%}$ assuming that the object temperature $T_{o b}=323$ $\mathrm{K}\left(50{ }^{\circ} \mathrm{C}\right)$. The values of the input variables of the model (1) should be assumed according to the table 2. The standard uncertainties of the input variables of the model (1) should be assumed according to the table 3 .

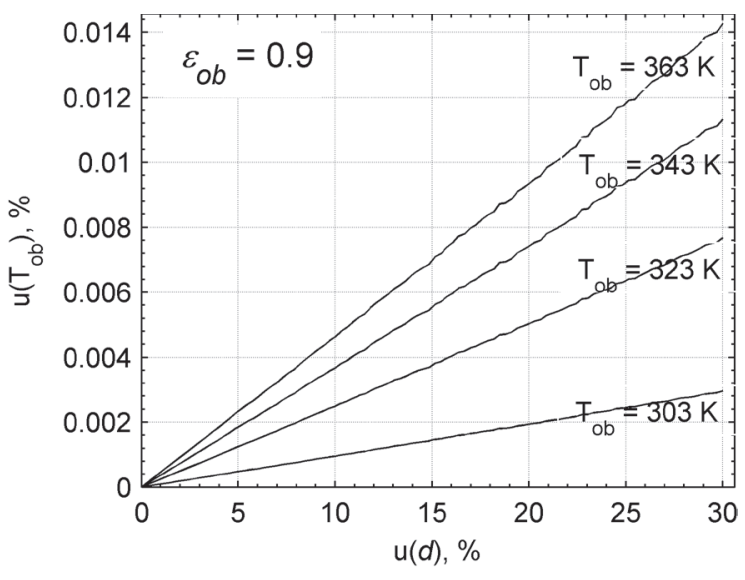

Fig. 7. Component of the relative standard uncertainty associated with the camera-to-object distance $d$. Results of simulations for data collected in tab. 1 .

Tab. 2: The values of the input variables of the model (1) assumed for the calculations of the combined standard uncertainty

\begin{tabular}{|c|c|c|c|c|}
\hline$\varepsilon_{o b}$ & $\begin{array}{c}T_{o} \\
(\mathrm{~K})\end{array}$ & $\begin{array}{c}T_{a t m} \\
(\mathrm{~K})\end{array}$ & $\omega$ & $\begin{array}{c}d \\
(\mathrm{~m})\end{array}$ \\
\hline 0,4 & 293 & 293 & 0,5 & 10 \\
0,9 & & \\
\hline
\end{tabular}

Tab. 3: Standard uncertainties of the input variables of the model (1) assumed for the calculations of the combined standard uncertainty

\begin{tabular}{|c|c|c|c|c|}
\hline$u\left(\varepsilon_{o b}\right)$ & $\begin{array}{c}u\left(T_{o}\right) \\
(\mathrm{K})\end{array}$ & $\begin{array}{c}u\left(T_{a t m}\right) \\
(\mathrm{K})\end{array}$ & $u(\omega)$ & $\begin{array}{c}u(d) \\
(\mathrm{m})\end{array}$ \\
\hline $\begin{array}{c}0,09 \\
(10 \%)\end{array}$ & 9 & 9 & 0,05 & 1 \\
0,04 & $(3 \%)$ & $(3 \%)$ & $(10 \%)$ & $(10 \%)$ \\
$(10 \%)$ & & & & \\
\hline
\end{tabular}

\section{Solution}

To solve the task from the exercise 7 , the simulations using data from tables 2 and 3 were carried out. The calculations were made with the program for simulations of the combined standard uncertainty. The screen shot of the program is presented in Fig. 8. The normalized histogram of the probability density function $\mathrm{g}\left(T_{o b}\right)$ of output variable $T_{o b}$ of model (1) is presented in Fig. 9. The ends of the $95 \%$ coverage interval are marked by vertical lines. The function $\mathrm{g}\left(T_{o b}\right)$ was evaluated with the method for the propagation of distributions and Monte Carlo simulations described in detail in [4]. In simulations the uniform distributions of the input variables were assumed. The value of the combined standard uncertainty $u_{c}\left(T_{o b}\right)$ of the object temperature for the input variable estimates collected in table 2 and the standard uncertainties collected in table 3 , was equal to $u_{c}\left(T_{o b}\right)=2,9 \mathrm{~K}(0,9 \%$ of the expected value $)-$ see Fig. 8.

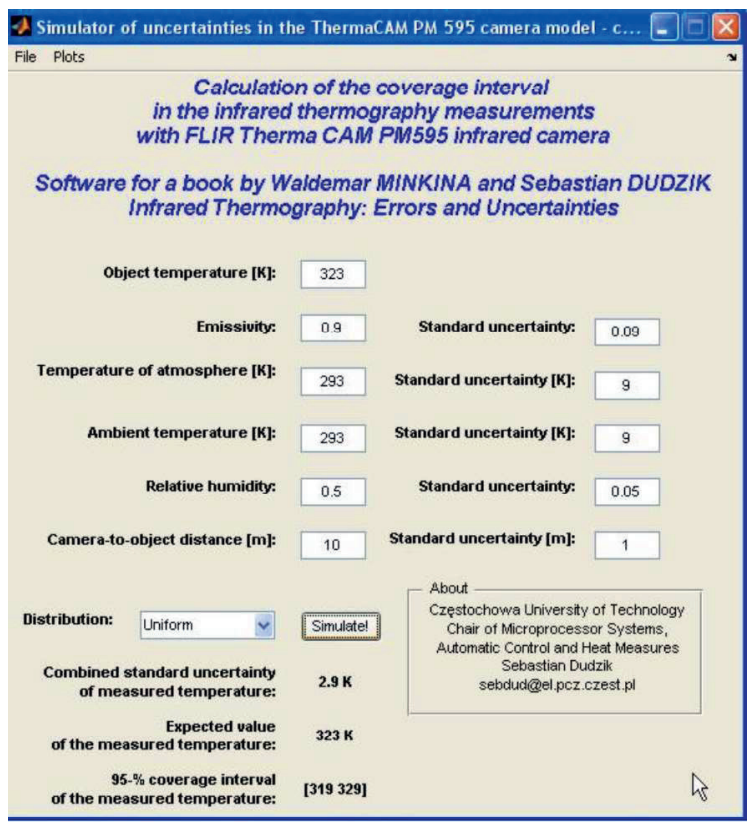

Fig. 8. The main window screen shot of the program used for the calculations of the combined standard uncertainty in the temperature measurements with the FLIR ThermaCAM PM595 infrared camera.

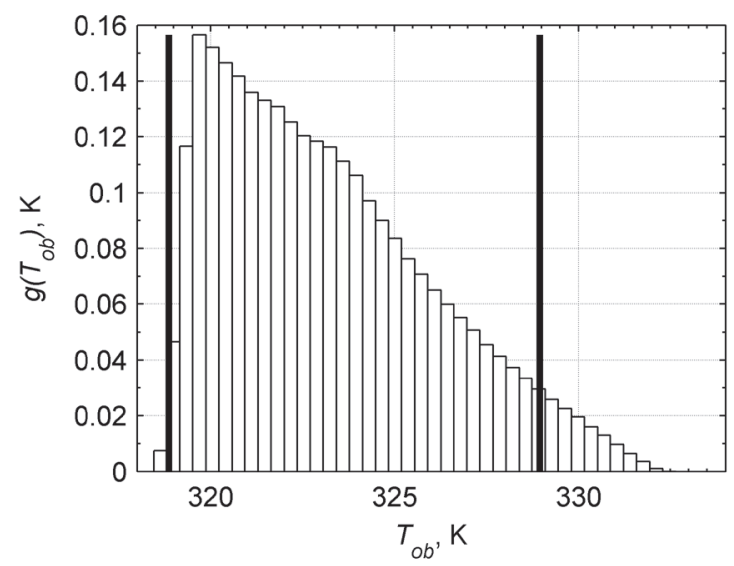

Fig. 9. The normalized histogram of the probability density function $g\left(T_{o b}\right)$ of output variable $T_{o b}$ of model (1). Results of simulations for data collected in tab. 2 and 3 (Exercise 7).

Calculations were carried out for $\varepsilon_{o b}=0,9$ and $T_{o b}=323 \mathrm{~K}\left(50{ }^{\circ} \mathrm{C}\right)$. The width of the $95 \%$ coverage interval for data from Exercise 7 can be read from Fig. 9. The shortest width is equal 
to $10 \mathrm{~K}$. The limits of the $95 \%$ coverage intervals read from Fig. 8 is $I_{95 \%}=[319,329] \mathrm{K}$.

\section{Exercise 8}

The temperature measurement was conducted using the FLIR ThermaCAM PM595 infrared camera. The emissivity of the investigated object was equal to $\varepsilon_{o b}=0,4$. Calculate the combined standard uncertainty $u_{c}\left(T_{o b}\right)$ and evaluate the $95 \%$ coverage interval $I_{95}$ assuming that the object temperature $T_{o b}=323$ $\mathrm{K}\left(50{ }^{\circ} \mathrm{C}\right)$. The values of the input variables of the model (1) should be assumed according to the table 2. The standard uncertainties of the input variables of the model (1) should be assumed according to the table 3 .

\section{Solution}

To solve the task from the exercise 8 , the simulations using data from tables 2 and 3 were carried out. The normalized histogram of the probability density function $\mathrm{g}\left(T_{o b}\right)$ of output variable $T_{o b}$ of model (1) is presented in Fig. 10. The ends of the $95 \%$ coverage interval are marked by vertical lines. In simulations the uniform distributions of the input variables were assumed. The value of the combined standard uncertainty $u_{c}\left(T_{o b}\right)$ of the object temperature for the input variable estimates collected in table 2 and the standard uncertainties collected in table 3 , was equal to $u_{c}\left(T_{o b}\right)=11 \mathrm{~K}(3,4 \%$ of the expected value). This value was read from the window analogous to the screen shot presented in Fig. 8.

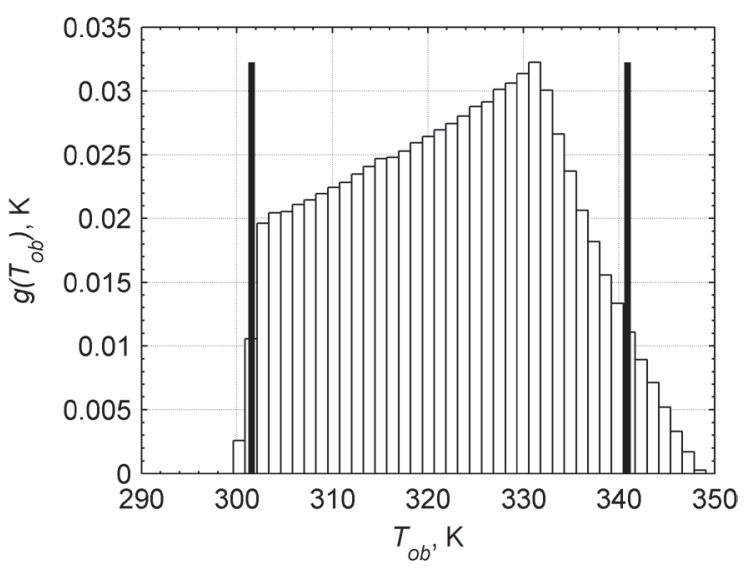

Fig. 10. The normalized histogram of the probability density function $g\left(T_{o b}\right)$ of output variable $T_{o b}$ of model (1). Results of simulations for data collected in tab. 2 and 3 (Exercise 8).

Calculations were carried out for $\varepsilon_{o b}=0,4$ and $T_{o b}=323 \mathrm{~K}\left(50{ }^{\circ} \mathrm{C}\right)$. The width of the $95 \%$ coverage interval for data from Exercise 8 can be read from Fig. 10. The shortest width is equal to $39 \mathrm{~K}$. The limits of the $95 \%$ coverage intervals read from Fig. 10 is $I_{95 \%}=[302,341] \mathrm{K}$.

\section{Conclusions}

In this work, the eight exercises of numerical calculations were presented. The purpose of calculations was an estimation of the thermographic measurement accuracy with in the presence of the systematic interactions. The model of the infrared temperature measurement was presented as well. Furthermore, the methodology of the $95 \%$ coverage in thermographic measurements interval was described. Summing up the results of the numerical calculations conducted in exercises the following conclusions can be drawn:

- The greatest impact on the combined standard uncertainty in thermographic measurements has a component associated with the object emissivity $\varepsilon_{o b}$

- The component associated with the object emissivity $\varepsilon_{o b}$ strongly depends on the temperature of investigated object $T_{o b}$

- Due to the complex and nonlinear model (1), the best method for the estimation of the combined standard uncertainty and the $95 \%$ coverage interval is the method for the propagation of distributions described in [4].

\section{References}

[1] Minkina W., Dudzik S.: „Infrared Thermography Errors and Uncertainties", John Wiley \& Sons Ltd, Chichester, (2009) ISBN 978-0-470-74718-6;

[2] Minkina W., Dudzik S., Gryś S.: „Errors of thermographic measurements - exercises" Proceedings of 10th International Conference on Quantitative Infrared Thermography (QIRT'2010) 27-30.07.2010 r., Quebec (Canada), organization: Université Laval, Electrical and Computer Engineering Department, pp. 503-509, ISBN 978-2-9809199-1-6;

[3] "Guide to the Expression of uncertainty in Measurement", ISO/TAG, editions: 1995, $1999 \mathrm{r}$.

[4] „Evaluation of measurement data - Supplement 1 to the Guide to the expression of uncertainty in measurement - Propagation of distributions using a Monte Carlo method", (2008), Joint Committee for Guides in Metrology.

[5] Dudzik S., Minkina W.: „Application of the numerical method for the propagation of distributions to the calculation of coverage intervals in the thermovision measurements", 9th International Conference on Quantitative Infrared Thermography QiRT, Kraków, (2008) July 2-5, pp. 179-184;

[6] Minkina W., Dudzik S.: „Simulation analysis of uncertainty of infrared camera measurement and processing path", Measurement, Vol. 39, (2006) Issue 8, pp. 758-763;

[7] Toolkit IC2, Dig 16 „Developers Guide” 1.00 AGEMA 550/570. 